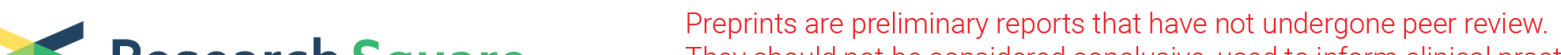 Research Square They should not be considered conclusive, used to inform clinical practice, or referenced by the media as validated information.
}

\section{High CS Expression Leads to Poor Prognosis Associated with Immune Invasion of Lung Adenocarcinoma}

\section{Ran Feng}

Affiliated Hospital of Nantong University, Nantong , Jiangsu

\section{Shihao Hong}

Medical School, Institute of Reproductive Medicine, Nantong University, Nantong

Tingting Bian

Affiliated Hospital of Nantong University, Nantong , Jiangsu

Xue Gu

Affiliated Hospital of Nantong University, Nantong , Jiangsu

Miaosen Zheng

Affiliated Hospital of Nantong University, Nantong , Jiangsu

Jian Liu

Affiliated Hospital of Nantong University, Nantong , Jiangsu

Yifei Liu ( $\nabla$ ntdxliuyifei@sina.com )

Affiliated Hospital of Nantong University, Nantong , Jiangsu

\section{Research Article}

Keywords: lung adenocarcinoma, CS, immunoinfiltrating, prognosis.

Posted Date: March 7th, 2022

DOI: https://doi.org/10.21203/rs.3.rs-1406141/v1

License: (c) (i) This work is licensed under a Creative Commons Attribution 4.0 International License. Read Full License 


\section{Abstract \\ Background}

Citrate synthase (CS) is the initial rate-limiting enzyme in tricarboxylic acid cycle. We found that its expression is up-regulated in many cancers. Previous studies on the relationship between the expression of CS and (LUAD) have not been reported till date.

\section{Methods}

we systematically analyzed several online databases, such as TCGA and GEO database, TIMER database, Kaplan Meier plotter, Ualcan database and Linkedomics database.

\section{Results}

we found that it was highly expressed in patients with LUAD. The high expression of CS caused poor prognosis in LUAD patients, and it was associated with some clinical traits. This indicates that it may be an independent prognostic factor. At the same time, we found that high CS expression was associated with the decline of immune-infiltrating B cells. While exploring the mechanism, we found that highly expressed CS mainly interacts with ATP5B and KPNB1 proteins. Moreover, CS is highly expressed in regulating the cell cycle, P53 binding site and oxygen binding pathways.

\section{Conclusions}

Our results confirmed CS was associated with LUAD and affected immune cell infiltration in LUAD. This provided a basis for further use of CS as a new therapeutic target and prognostic indicator of patients with LUAD.

\section{Introduction}

The morbidity and mortality of lung cancer have increased tremendously and it has become a very common disease today. In 2018, GLOBOCAN reported that 2.09 million new cases and 1.76 million deaths related to lung cancer. Compared to 2012, nearly 200,000 more new cases and nearly 160,000 more deaths occurred due to lung cancer in 2018 (1).Histologically, lung cancer can be differentiated into two types: non-small cell lung cancer(NSCLC) and small cell lung cancer (SCLC). Furthermore, LUAD is the most important subtype of $\operatorname{NSCLC}(2,3)$.In the past decade, the treatment of NSCLC has advanced tremendously. Currently, surgery, radiotherapy, chemotherapy, tumor immunity and driver mutations are the most conventional methods used for treating NSCLC cases. Despite these efforts, the 5-year survival rate of LUAD is still very low (4).Therefore, a new method of immunotherapy must be developed for the treatment of LUAD. Moreover, sophisticated diagnostic techniques must be used to detect LUAD at an early stage. 
A close relationship exists between tumor and its surrounding microenvironment. The surrounding immune infiltrating cells have different functions and can affect the tumor(5) After reviewing many literature studies of bioinformatics, we found that CS is a potential prognostic marker of LUAD.CS is an enzyme that plays a pivotal role in tricarboxylic acid cycle. In this process, CS catalyzes the condensation of acetyl-CoA and oxaloacetic acid to produce citric acid. Moreover, it is an important regulatory enzyme that facilitates the formation of nicotinamide adenine dinucleotide (NADH) and flavine adenine dinucleotide (FADH)2. These compounds are used for oxidative phosphorylation(6). Recently, the development of a variety of cancers was associated with changes in the expression of CS (7-10). For example, CS was highly expressed in cases of human prostate, pancreatic and ovarian cancers $(7,8,10)$. However, it has also been reported that the malignancy of cervical cancer could be aggravated with the knockdown of CS. In this case, the proliferation of tumor cells and metastasis would increase (9). However, we do not yet know the effect of CS in LUAD.

\section{Results}

\subsection{The expression of CS in different human cancers}

In this study, we evaluated the difference between the expression levels of mRNA and CS gene in different tumor tissues and adjacent tissues. The result showed that the expression of CS gene was up-regulated in the most tumors, such as breast cancer (BRCA), cholangiocarcinoma (CHOL), colon adenocarcinoma (COAD), esophageal carcinoma (ESCA), head and neck squamous cell carcinoma (HNSCC), human papillomavirus (HPV)-positive squamous cell carcinoma, kidney chromophobe $(\mathrm{KICH})$, kidney renal clear cell carcinoma (KIRC), liver hepatocellular carcinoma (LIHC), lung squamous cell carcinoma (LUSC), prostate adenocarcinoma (PRAD), stomach adenocarcinoma (STAD) and LUAD(Fig. 1A). In TCGA database, the expression of CS in tumor tissues was higher compared with para-cancer tissues (Fig. 1B). Compared to the adjacent tissue, the expression level of CS was higher in tumor tissue (Fig. 1C) Meanwhile, we also collected clinical samples for immunohistochemical verification. The results indicated that CS was highly expressed in LUAD tissues (Fig. 1D). Thus, CS is highly expressed in patients with LUAD.

\subsection{The relationship between the expression of CS and clinical characters}

In this experiment, we investigated the correlation between the expression of CS and clinical characters of LUAD patients. Using the Ualcan datebase, we found that the expression of CS was significantly elevated in LUAD patients with the following clinical features: advanced age, lymph node metastasis, male gender, T3 or T4, frequent smoking and TP53 mutation (Fig. 2A-F). In TCGA database, the results of logistic regression analysis showed that the expression of CS was different in the following groups: T2 vs T1 $(P<0.014), T 3$ vs T1 $(P<0.005)$ and male vs female $(P<0.031)$. Meanwhile, there was no difference in the expression of CS with following clinical parameters: age, stage, lymph node metastasis and distant metastasis (Table 1). In order to further analyze the relationship between CS and clinicopathological parameters of patients with LUAD, we collected and chemically stained the immunohistochemical microarray of patients with LUAD. Based on the staining index, we divided the samples into two groups. The results indicated significant differences in the age, gender, stage, tumor size, the presence of lymph node metastasis and smoking habits 
of LUAD patients with highly expressed CS and low expressed CS (Table 2). Thus, the expression of CS is closely related to the clinical characteristics of LUAD patients.

Table 1

The relationship between CS and clinicopathological parameters

\begin{tabular}{|llll|}
\hline Clinical parameters & Total(N) & $\begin{array}{l}\text { Odds ratio in CS } \\
\text { expression }\end{array}$ & P-Value \\
\hline Age $(\leq 60$ vs $>60)$ & 468 & $0.961(0.651-1.419)$ & 0.843 \\
\hline Gender(male vs female) & 487 & $1.489(1.037-2.141)$ & 0.031 \\
\hline Stage(II vs I) & 374 & $0.932(0.595-1.459)$ & 0.758 \\
\hline Stage(III vs I) & 341 & $0.889(0.534-1.475)$ & 0.648 \\
\hline Stage(IV vs I) & 327 & $1.378(0.595-3.305)$ & 0.458 \\
\hline Tumor size (T2 vs T1) & 423 & $1.653(1.109-2.473)$ & 0.014 \\
\hline Tumor size (T3 vs T1) & 205 & $2.789(1.379-5.856)$ & 0.005 \\
\hline Tumor size (T4 vs T1) & 183 & $1.301(0.491-3.403)$ & 0.588 \\
\hline Lymph node metastasis (positive vs negative) & 474 & $1.267(0.863-1.864)$ & 0.227 \\
\hline Distant metastasis(positive vs negative) & 358 & $1.730(0.748-4.224)$ & 0.209 \\
\hline
\end{tabular}


Table 2

The relationship between CS expression and clinicopathological parameters in lung adenocarcinoma tissue chips

\begin{tabular}{|c|c|c|c|c|}
\hline \multirow[t]{2}{*}{ Clinicopathological parameters } & \multirow[t]{2}{*}{ All cases } & \multicolumn{2}{|c|}{ CS expression } & \multirow[t]{2}{*}{ P-value } \\
\hline & & Low & High & \\
\hline $\operatorname{Age}(\leq 60)$ & 83 & 15 & 68 & 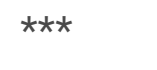 \\
\hline Age $(>60)$ & 129 & 27 & 102 & $\star \star *$ \\
\hline Gender(Male) & 131 & 24 & 107 & 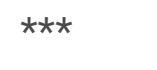 \\
\hline Gender(Female) & 81 & 18 & 63 & $\star \star \star$ \\
\hline Nonsmoker & 167 & 35 & 132 & 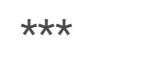 \\
\hline Smoker & 45 & 7 & 38 & 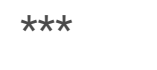 \\
\hline Stage $(I+I I)$ & 155 & 29 & 126 & $\star \star *$ \\
\hline Stage(III+ IV) & 57 & 13 & 44 & 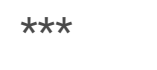 \\
\hline T classification $(\mathrm{T} 1+\mathrm{T} 2)$ & 177 & 21 & 156 & $\star \star \star ~$ \\
\hline T classification $(\mathrm{T} 3+\mathrm{T} 4)$ & 35 & 7 & 28 & $\star \star \star *$ \\
\hline $\mathrm{N}$ classification(N0) & 117 & 21 & 96 & $\star \star \star$ \\
\hline $\mathrm{N}$ classification(N1 + 2) & 95 & 21 & 74 & $\star \star \star ~$ \\
\hline
\end{tabular}

\subsection{The survival significance of CS and its independent prognostic factor}

Based on KM survival curves, we observed that overexpression of CS was associated with poor prognosis (Fig. 3A). Homoplastically, in GSE14818, we established that high-risk groups had poor prognosis as compared to low-risk groups (Fig. 3B). In TCGA database, the optimal cutoff value was selected according to the expression of $\mathrm{CS}$, the samples were divided into high and low expression groups: the prognosis of high CS expression group was poor (Fig. 3C). In TCGA and GEO databases, Cox regression analysis was performed to investigate the relationship between CS expression and clinicopathological parameters. Univariate analysis showed that all the clinicopathological parameters (age, gender, disease stage, tumor size, lymph node metastasis and distant metastasis) were correlated with the prognosis and survival of patients with CS expression. Multivariate analysis showed that age, disease stage, tumor size, lymph node metastasis, and CS expression had an independent prognostic value (Table 3, Fig. 3D). Thus, CS may be used as an independent prognostic factor in the diagnosis of LUAD. 
Table 3

Univariate and multivariate analysis of CS and clinical traits in patients with lung adenocarcinoma

\begin{tabular}{|lllll|}
\hline Clinicopathologic parameters & \multicolumn{3}{l}{ Univariate analysis } & \multicolumn{3}{l|}{ Multivariate analysis } \\
\hline & $\mathrm{HR}(95 \% \mathrm{Cl})$ & P-value & $\mathrm{HR}(95 \% \mathrm{Cl})$ & P-value \\
age & $1.018(1.007-1.028)$ & $<0.001$ & $1.026(1.015-1.037)$ & $<0.001$ \\
gender & $1.314(1.072-1.610)$ & 0.008 & $1.211(0.987-1.485)$ & 0.066 \\
stage & $1.509(1.400-1.626)$ & $<0.001$ & $1.296(1.080-1.555)$ & 0.005 \\
T & $1.843(1.591-2.135)$ & $<0.001$ & $1.361(1.173-1.581)$ & $<0.001$ \\
M & $2.201(1.797-2.696)$ & $<0.001$ & $1.325(0.838-2.093)$ & 0.228 \\
\hline N & $1.981(1.749-2.244)$ & $<0.001$ & $1.507(1.274-1.783)$ & $<0.001$ \\
\hline CS & $1.021(1.010-1.031)$ & $<0.001$ & $1.017(1.005-1.030)$ & 0.006 \\
\hline
\end{tabular}

\subsection{The relationship between the expression of CS and TIICs and their marker genes}

Several evidences prove that TIICs change with changes in tumor tissues(11-13). Therefore, TIICs are closely associated with the diagnosis and prognosis of LUAD patients. To ascertain whether CS affected the prognosis of LUAD patients by affecting TIICs, we obtained samples from TCGA database. We found that the expression of CS was negatively correlated with the following kinds of immune cells: activated B cell, activated dendrite cell, central memory CD4 T cell, eosinophils, mast cell, monocyte, T follicular helper cell and Type 1 helper cell. Meanwhile, activated CD4 T cell and CD56 dim natural killer cell also evinced high infiltration when CS was highly expressed (Fig. 4A). In the TIMER database, CS gene was strongly correlated with CD8 + T cells $(r=0.137, P=2.47 e-3)$ and neutrophils $(r=0.129, P=4.38 e-3)$ (Fig. 4B). Moreover, we found that a good prognosis with low CS expression with highly infiltrated B cells and dendritic cells (Fig. 4C). Furthermore, we elucidated the relationship between CS and immune invasion gene in TIMER database. We found that some marker genes were positively correlated with the expression of CS in following cell types: tumor-associated macrophages (TAM), macrophage cell, CD8 + T cell, NK cell, dendritic cell, helper T cell, Tfh cell, Treg cell and T cell exhaustion. Moreover, it also showed that CS expression was negatively associated with T cell(general), B cell and dendritic cell marker genes (Table 4). To further confirmed our finding, we scoured the GEPIA database and found that CS expression was positively correlated with these genes: CD8 + T cell, TAM, M1 Macrophage, M2 Macrophage and Treg. At the same time, it was negatively correlated with T cell marker gene and B cell marker gene (Table 5). Thus, CS gene may be an immune-related prognostic factor. 
Table 4

The relation between CS expression and TIICs is in TIMER

\begin{tabular}{|c|c|c|c|c|c|}
\hline Description & Marker genes & None Cor & $\mathbf{P}$ & Purity Cor & $\mathbf{P}$ \\
\hline \multirow[t]{3}{*}{ CD8 + T cell } & CD8A & 0.091 & * & 0.138 & ** \\
\hline & $\mathrm{CD} 8 \mathrm{~B}$ & 0.036 & 0.41 & 0.057 & 0.207 \\
\hline & CD45(PTPRC) & 0.112 & * & 0.18 & $\star \star \star *$ \\
\hline \multirow[t]{3}{*}{ T cell (general) } & CD3D & -0.064 & 0.146 & -0.031 & -0.49 \\
\hline & CD3E & 0.021 & 0.638 & 0.079 & 0.0799 \\
\hline & $\mathrm{CD} 2$ & 0.011 & 0.798 & 0.062 & 0.172 \\
\hline \multirow[t]{4}{*}{ B cell } & CD19 & -0.074 & 0.094 & -0.042 & 0.356 \\
\hline & CD79A & -0.088 & * & -0.051 & 0.257 \\
\hline & CD27 & -0.059 & 0.178 & -0.015 & 0.743 \\
\hline & CD2O & -0.027 & 0.539 & 0.008 & 0.86 \\
\hline \multirow[t]{3}{*}{ Monocyte } & CD14 & -0.027 & 0.539 & 0.008 & 0.86 \\
\hline & CD86 & 0.042 & 0.341 & 0.081 & 0.073 \\
\hline & CD115 (CSF1R) & 0.064 & 0.148 & 0.11 & * \\
\hline \multirow[t]{2}{*}{ TAM } & CD68 & 0.086 & 0.0519 & 0.119 & ** \\
\hline & CD118(LIFR) & 0.174 & $\star \star \star *$ & 0.173 & $\star \star \star *$ \\
\hline \multirow[t]{3}{*}{ M1 Macrophage } & INOS (NOS2) & 0.135 & ** & 0.145 & ** \\
\hline & IRF5 & 0.05 & 0.26 & 0.067 & 0.139 \\
\hline & COX2(PTGS2) & 0.051 & 0.251 & 0.044 & 0.331 \\
\hline \multirow[t]{2}{*}{ M2 Macrophage } & CD163 & 0.206 & 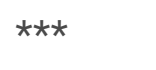 & 0.206 & $\star \star \star \star$ \\
\hline & CD206(MRC1) & 0.052 & 0.238 & 0.083 & 0.0643 \\
\hline \multirow[t]{4}{*}{ Neutrophil } & CD66b (CEACAM8) & -0.03 & 0.501 & -0.02 & 0.662 \\
\hline & CD11b (ITGAM) & 0.061 & 0.168 & 0.106 & * \\
\hline & CD15 (FUT4) & 0.205 & $\star \star \star *$ & 0.219 & $\star \star \star$ \\
\hline & CCR7 & -0.044 & 0.317 & -0.001 & 0.977 \\
\hline \multirow[t]{3}{*}{ Natural killer cell } & KIR2DL1 & 0.044 & 0.317 & 0.054 & 0.227 \\
\hline & KIR2DL3 & 0.095 & * & 0.117 & ** \\
\hline & KIR2DL4 & 0.188 & 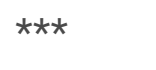 & 0.215 & $\star \star \star ~$ \\
\hline
\end{tabular}

Ns: no significance; ${ }^{\star} \mathrm{P}<0.05 ;{ }^{* * \mathrm{P}}<0.01 ; * \star * \mathrm{P}<0.001$. 


\begin{tabular}{|c|c|c|c|c|c|}
\hline Description & Marker genes & None Cor & $\mathbf{P}$ & Purity Cor & $\mathbf{P}$ \\
\hline & KIR3DL1 & 0.071 & 0.107 & 0.089 & * \\
\hline & KIR3DL2 & 0.111 & * & 0.145 & $\star \star$ \\
\hline & KIR3DL3 & 0.06 & 0.172 & 0.08 & 0.0746 \\
\hline & KIR2DS4 & 0.095 & * & 0.121 & $\star \star$ \\
\hline & CD335 (NCR1) & 0.184 & 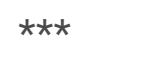 & 0.219 & $\star \star \star *$ \\
\hline \multirow[t]{4}{*}{ Dendritic cell } & BDCA-1 (CD1C) & -0.227 & $\star \star \star \star$ & -0.213 & $\star \star \star$ \\
\hline & BDCA-3 (CD141) & -0.076 & 0.0867 & -0.06 & 0.186 \\
\hline & BDCA-4 (NRP1) & 0.195 & $\star \star \star \star$ & 0.215 & $\star \star \star$ \\
\hline & CD11c (ITGAX) & 0.073 & 0.0969 & 0.124 & $\star *$ \\
\hline \multirow[t]{5}{*}{ Th1 } & T-bet (TBX21) & 0.075 & 0.0889 & 0.118 & ** \\
\hline & STAT4 & 0.042 & 0.342 & 0.075 & 0.0975 \\
\hline & IFN-y (IFNG) & 0.15 & 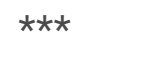 & 0.189 & $\star \star \star *$ \\
\hline & TNF-a (TNF) & 0.048 & 0.279 & 0.087 & 0.0548 \\
\hline & STAT1 & 0.345 & $\star \star \star \star ~$ & 0.391 & $\star \star \star *$ \\
\hline \multirow[t]{3}{*}{ Th2 } & GATA3 & 0.032 & 0.472 & 0.074 & 0.0989 \\
\hline & STAT5A & 0.071 & 0.107 & 0.12 & $\star \star$ \\
\hline & STAT6 & 0.312 & 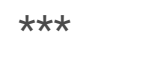 & 0.304 & $\star \star \star *$ \\
\hline \multirow[t]{5}{*}{ Tfh } & BCL6 & 0.078 & 0.0766 & 0.089 & * \\
\hline & IL21 & 0.221 & $\star \star \star *$ & 0.248 & $\star \star \star *$ \\
\hline & STAT3 & 0.316 & 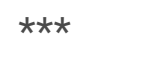 & 0.325 & $\star \star \star *$ \\
\hline & IL17A & 0.042 & 0.34 & 0.051 & 0.255 \\
\hline & RORyt(IL17A) & 0.042 & 0.34 & 0.051 & 0.255 \\
\hline \multirow[t]{5}{*}{ Treg } & FOXP3 & 0.117 & ** & 0.176 & $\star \star \star$ \\
\hline & CD25 (IL2RA) & 0.228 & 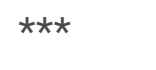 & 0.283 & $\star \star \star *$ \\
\hline & CCR8 & 0.212 & 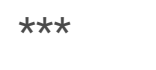 & 0.274 & $\star \star \star *$ \\
\hline & TGF $\beta$ (TGFB1) & 0.013 & 0.765 & 0.045 & 0.317 \\
\hline & STAT5B & 0.243 & $\star \star \star *$ & 0.258 & $* \star \star$ \\
\hline T cell exhaustion & PD-1 (PDCD1) & 0.093 & * & 0.146 & ** \\
\hline
\end{tabular}

Ns: no significance; ${ }^{\star} \mathrm{P}<0.05 ;{ }^{\star \star} \mathrm{P}<0.01$; ${ }^{* \star *} \mathrm{P}<0.001$. 


\begin{tabular}{|clllll|}
\hline Description & Marker genes & None Cor & P & Purity Cor & P \\
\hline CTLA4 & 0.087 & $*$ & 0.146 & $* *$ \\
\hline LAG3 & 0.101 & $*$ & 0.145 & $* \star$ \\
\hline TIM-3 (HAVCR2) & 0.034 & 0.436 & 0.073 & 0.106 \\
\hline GZMB & 0.163 & $* * *$ & 0.208 & $* * *$ \\
\hline Ns: no significance; ${ }^{*} \mathrm{P}<0.05 ; * * \mathrm{P}<0.01 ; * * * \mathrm{P}<0.001$. & & & \\
\hline
\end{tabular}

Table 5

The relation between CS expression and TIICs is in GEPIA

\begin{tabular}{|c|c|c|c|c|c|}
\hline Description & Marker genes & Tumor $\mathbf{R}$ & $\mathbf{P}$ & Normal R & $\mathbf{P}$ \\
\hline \multirow[t]{3}{*}{$\mathrm{CD} 8+\mathrm{T}$ cell } & CD8A & 0.025 & 0.59 & 0.2 & 0.13 \\
\hline & $\mathrm{CD} 8 \mathrm{~B}$ & 0.03 & 0.51 & 0.12 & 0.37 \\
\hline & CD45 & 0.1 & * & 0.5 & $\star \star \star *$ \\
\hline \multirow[t]{3}{*}{ T cell (general) } & CD3D & -0.15 & $\star \star \star$ & -0.16 & 0.24 \\
\hline & CD3E & -0.028 & 0.53 & 0.25 & 0.059 \\
\hline & $\mathrm{CD} 2$ & -0.014 & 0.76 & 0.089 & 0.5 \\
\hline \multirow[t]{3}{*}{ B cell } & CD19 & -0.16 & $\star \star \star$ & 0.065 & 0.62 \\
\hline & CD79A & -0.21 & $\star \star \star$ & 0.06 & 0.65 \\
\hline & CD27 & -0.19 & $\star \star \star$ & 0.13 & 0.32 \\
\hline \multirow[t]{2}{*}{ TAM } & CD68 & 0.13 & $\star \star \star$ & 0.3 & * \\
\hline & CD11B & 0.081 & 0.076 & 0.32 & * \\
\hline \multirow[t]{2}{*}{ M1 Macrophage } & IRF5 & 0.12 & $\star \star \star$ & 0.19 & 0.15 \\
\hline & PTGS2 & 0.033 & 0.47 & 0.2 & 0.14 \\
\hline \multirow[t]{2}{*}{ M2 Macrophage } & CD163 & 0.094 & * & 0.029 & 0.83 \\
\hline & CD206 & 0.044 & 0.34 & 0.44 & $\star \star \star$ \\
\hline \multirow[t]{4}{*}{ Treg } & FOXP3 & 0.08 & 0.08 & 0.28 & 0.033 \\
\hline & CCR8 & 0.16 & $\star \star \star$ & 0.15 & 0.26 \\
\hline & TGFB1 & 0.034 & 0.46 & 0.54 & $\star \star \star *$ \\
\hline & STAT5B & 0.26 & $\star \star \star$ & 0.71 & $\star \star \star$ \\
\hline
\end{tabular}




\subsection{Positive and negative genes and metabolic pathways associated with CS}

In order to unravel the biological functions of CS, we used LinkedOmics database and explored the coexpression genes of CS in LUAD tissues. In the LinkedOmics database, we found that 4212 gene (dark green dots) had a significantly negative relationship with CS. On the other hand, we found that 2948 genes (dark red dots) were positively associated with CS (Fig. 5A). The genes that showed a positive correlation with CS were as follows: ATP5B, BAZ2A, and DNAJC14 etc. The expression of CS showed a strong negative correlation with the expression of following genes: SEPW1, ZNF688, and C6orf225 (Fig. 5B-C). Gene enrichment analysis was related to CS in LinkedOmics database. By performing KEGG pathway analysis, we identified CS related genes in LinkedOmics database by performing GOCC pathway analysis, we performed CS related genes in LinkedOmics database. By performing GOBP pathway analysis, we identified CS related genes in LinkedOmics database (Fig. 5D-F).

\subsection{Construct PPI network of CS co-expressed genes}

Protein-protein interaction (PPI) network analysis was performed to identify the positive and negative genes associated with CS. Using Cytoscape visualization software, we obtained the interactivity map of positive and negative genes (Fig. 6A-B). Then the MCODE plugin of Cytoscape software was used to decipher the subnetwork of CS-related positive and negative genes that formed clust1 and clust2. Clust1 showed that CS related interacting proteins were mainly as follows: FOXM1, ESPL1, HSPD1, MKI67, PA2G4, RACGAP1, NCAPD2, SHMT2, NOP2, HNRNPA1L2, PLK3, CKAP5, TIMELESS, KIF20B, PRMT2 and CDK2. Clust2 showed that CS related interacting proteins were mainly as follows: PDCD11, POLR3A, ATP5B, KPNB1, XPOT, MARS, UTP20, NOLC1, DDX21 and GCN1L1 (Fig. 6C-D). Thus, CS interacts with these proteins and may be involved in pathways that influence their regulation

\subsection{Gene sets enriched in CS expression}

To clarify whether LUAD was regulated by CS, we performed KEGG and GO enrichment analysis on CS. The result shows the KEGG and GO pathways associated with an overexpression of CS (Table 6). The KEGG related pathways: cell cycle, oocyte meiosis, RNA degradation, lysine degradation, autoimmune rejection, autoimmune thyroid disease, and hematopoietic cell lineage (Fig. 7A). The GO related pathways: P53 binding sites, oxygen binding, double stranded RNA that binds the negative end of ATP-dependent microtubule, motor activity, interleukin 5 production, nuclear mrna output, mrna transport, nuclear output, structural composition of nuclear pores, RNA decompenase activity, pre-mrna 5 splice site binding, sperm midsection, nuclear periphery, motor cilia, complex histone methyltransferase, fibrous center, and cytoplasmic particle pressure (Fig. 7B-D). 
Table 6

The highly expressed CS enrichment pathway was analyzed in KEGG and GO

\begin{tabular}{|llll|}
\hline Gene set name(High expression) & NES & $\begin{array}{l}\text { NOM } \\
\text { p_X0002_val }\end{array}$ & $\begin{array}{l}\text { FDR } \\
\text { q_X002__val }\end{array}$ \\
\hline KEGG_CELL_CYCLE & 2.4030907 & 0 & 0 \\
\hline KEGG_OOCYTE_MEIOSIS & 2.3234177 & 0 & $2.71 \mathrm{E}-04$ \\
\hline KEGG_LYSINE_DEGRADATION & 2.3286157 & 0 & $3.62 \mathrm{E}-04$ \\
\hline KEGG_RNA_DEGRADATION & 2.2365367 & 0.002178649 & $7.63 \mathrm{E}-04$ \\
\hline GOBP_NUCLEAR_EXPORT & 2.66489 & 0 & 0 \\
\hline GOBP_NUCLEAR_TRANSPORT & 2.6054733 & 0 & 0 \\
\hline GOBP_MRNA_TRANSPORT & 2.5735826 & 0 & 0 \\
\hline GOBP_MRNA_EXPORT_FROM_NUCLEUS & 2.5763261 & 0 & 0 \\
\hline GOBP_TELOMERE_ORGANIZATION & 2.5919397 & 0 & 0 \\
\hline GOMF_RNA_HELICASE_ACTIVITY & 2.4900894 & 0 & 0 \\
\hline GOMF_P53_BINDING & 2.3149304 & 0 & $1.55 \mathrm{E}-04$ \\
\hline GOMF_3_5_EXONUCLEASE_ACTIVITY & 2.320904 & 0 & $1.93 \mathrm{E}-04$ \\
\hline GOMF_STRUCTURAL_CONSTITUENT_OF_NUCLEAR_PORE & 2.3729482 & 0 & $1.57 \mathrm{E}-04$ \\
\hline GOMF_DNA_HELICASE_ACTIVITY & 2.3203266 & 0 & $1.82 \mathrm{E}-04$ \\
\hline GOMF_DOUBLE_STRANDED_RNA_BINDING & 2.3717282 & 0 & $1.37 \mathrm{E}-04$ \\
\hline GOCC_CYTOPLASMIC_STRESS_GRANULE & 2.4579816 & 0 & 0 \\
\hline GOCC_FIBRILLAR_CENTER & 2.4424264 & 0 & 0 \\
\hline GOCC_HISTONE_METHYLTRANSFERASE_COMPLEX & 2.3682127 & 0 & 0 \\
\hline GOCC_NUCLEAR_PERIPHERY & 2.4096656 & 0 & 0 \\
\hline GOCC_ATPASE_COMPLEX & 2.389002 & 0 & 0 \\
\hline
\end{tabular}

\section{Discussion}

In China, the prevalence of lung cancer is expected to increase by about 40\% between 2015 and 2030 (18). Although there have been advances in the diagnosis and treatment of LUAD, the survival rate of LUAD patients continues to be low due to poor prognosis. Therefore, extensive research must be conducted to improve the conventional treatment of immunotherapy. Presently, CS causeing changes in the tricarboxylic acid cycle has been observed frequently in human tumors (19). In the main reaction site of citric acid cycle, cancer can be very easily induced by abnormal metabolic enzymes and metabolites of mitochondria. This can adversely affect the functions of mitochondria and the metabolism of cancer (20). Moreover, CS is 
abnormally expressed in a variety of malignant tumors, such as ovarian cancer, pancreatic cancer, cervical cancer and kidney cancer $(7,9,21,22)$. Recently, immunotherapy has shown its efficacy in cancer treatment. We have identified the type of TIICs that were closely related to LUAD. (23). Therefore, the relationship between CS and TIICs is worth exploring.

Firstly, in the TIMER database and TCGA database. we confirmed that that mRNA of CS was significantly overexpressed in LUAD. The immunohistochemical results were similar to the expression of CS in TCGA database. Then we manifested the fact that the expression levels of CS were correlated with age, nodal metastasis status, gender, disease stages, smoking habits and TP53 mutation status in LUAD patients. By performing logistic regression analysis of CS expression and its clinical traits, we found that CS expression was closely related to gender and tumor size. Moreover, there was no significant difference between the expression of CS and age, stage, lymph node metastasis and whether there was distant metastasis. This occurred probably due to the deletion of some meaningless data. Meanwhile, the Kaplan-Meier plotter, TCGA database and GEO database established that patients with low CS expression had a better possibility of survival than those with high CS expression. By performing univariate and multivariate regression, we found that CS is an independent prognostic indicator. Therefore, CS can be considered as an effective therapeutic target in the prognosis of LUAD.

Previous studies have reported that a complex exists between the immune system and tumors. There are immune cells that kill tumors, and there are immune cells that promote the growth of tumors (24). CD4 + Treg cells promote the immunity of tumor tissues by performing immunosuppressive functions (25). B cells secrete antibodies and NK cells kill tumor cells (26). Infiltrating B cells exhibit their own anti-tumor effects in patients with lung cancer (27). By using CIBERSORT algorithm, we found that CS expression mainly affected T cells, especially central memory T cells, in patients with LUAD. In the TIMER database and GEPIA database. we found that a high CS expression led to the decline of B cells. Consequently, the prognosis was poor in patients with LUAD. Therefore, we suggest that CS intensifies the progression of LUAD by affecting TIICs in patients.

Therefore, CS is now considered as a potential prognostic target of LUAD, we do not know the underlying mechanism through which CS fortifies the progression of LUAD. In LinkedOmics database, we screened out the positive and negative genes related to CS and conducted PPI net analysis. We found CS interacts with ATP5B and KPNB1. Previous studies have found that KPNB1 promotes tumor growth by regulating cell cycle and apoptosis (28). Furthermore, ATP5B increases the content of ATP in cells, thereby promoting cell migration, invasion, and proliferation. (29). Therefore, we suspected that CS may affect the development of tumors by influencing the proteins that interact with CS. Then we analyzed KEGG and GO in TCGA database and found that CS expression was mainly related to cell cycle and oxygen binding. Previous studies have also reported that CS caused a decrease in the consumption of cellular oxygen and ATP production, mitochondrial respiratory chain affected the metabolism of energy. These events led to an oxidative damage of the mitochondria and affecting cell proliferation (30). Therefore, we could guess that CS may affect oxygen binding and cell cycle by influencing mitochondria. Cell proliferation promotes the development of LUAD. 
In conclusion, we proved that high CS expression contributes to the development and progression of LUAD. Moreover, we also elucidated the relationship between CS and TIICs. Therefore, CS may be a potential therapeutic target in lung adenocarcinoma.

\section{Materials And Methods}

\subsection{An analysis of TCGA and GEO database}

Firstly, gene and clinical data were retrieved from the TCGA database (https://genome-cancer.ucsc.edu/). Then the data were processed further with R software (version 4.0.3, R Foundation, Vienna, Austria) and Perl software (Strawberry version, Larry Wall, Los Angeles, CA, USA).

\subsection{An analysis of TIMER database}

TIMER database (https://cistrome.shinyapps.io/timer/) is a web page that displays the relationship between genes and immune infiltrating cells (14). Therefore, a series of analyses were conducted to determine the expression of CS in different tumors and to ascertain its relationship with tumor immunoinfiltrating cells. In the related module, the correlation between CS and immune infiltrating cells was established.

\subsection{Kaplan-Meier plotter analysis}

Using the Kaplan-Meier plotter (http://kmplot.com/ analysis/), we determined whether the presence of CS was useful for the survival of patients with LUAD. In this online analysis, log rank $p$ value $<0.05$ was considered to be statistically significant (15).

\subsection{An analysis of Ualcan database}

Ualcan (http://ualcan.path.uab.edu/index.html) is an effective cancer data mining site, which data is mainly based on the cKaplan-Meier plotter (http://kmplot.com/ analysis/), ancer-related data of TCGA database. It was used to analyze the expression of CS in patients with different clinical types of cancer.

\subsection{An Analysis of LinkedOmics Database}

LinkedOmics (http://www.linkedomics.org/login.php) is an online website that is used for analyzing target genes, positive and negative genes and the related enrichment pathways. In this experiment, we obtained heat maps of positive and negative genes that were related to target genes.

\subsection{Immunohistochemistry Analysis}

The organization is from affiliated Hospital of Nantong University and approved by the Ethics Committee of Affiliated Hospital of Nantong University (review number: 2021-L021). A tissue microarray was formed after performing the paraffin-embedding method. Briefly, slices of $3 \mu \mathrm{m}$ thickness were cut continuously from the wax block. Then they were baked at $70{ }^{\circ} \mathrm{C}$ for 2 hours. These sections were then dewaxed in $100 \%$ xylene for $10 \mathrm{~min}$. After they were rehydrated in gradient ethanol for 2 minutes. Finally, they were maintained in EDTA buffer $(\mathrm{pH}=9.0)$ for $20 \mathrm{~min}$. A primary antibody (diluted 1:400, Sigma Aldrich, St. Louis, Missouri, USA) was dropped into the sections and they were incubated at room temperature for 1 hour. Then the sections were covered with rabbit secondary antibody (1:400, ab96600; Abcam) and colored with diaminobenzidine (DAB). 
The sections were re-stained with hematoxylin. Based on the degree of staining, the sections were classified into $1,2,3$, and 4 point. The staining ranges of $0-25 \%, 26-50 \%, 51-75 \%$, and $76-100 \%$ were graded $1,2,3$, and 4 respectively. The final dyeing index was obtained by multiplying the dyeing degree score with the dyeing range score. If the staining index of the section was less than 2.75 , it was considered as negative. If the staining index of the section was greater than 2.75 , it was considered as positive. All the sections were graded by two qualified pathologists.

\subsection{CIBERSORT Analysis}

CIBERSORT (http://cibersort.stanford.edu/) is a website that is used for online analysis of gene expression, which changes with the distribution of peripheral immune cells(16). In this experiment, we divided 497 samples into high and low expression groups of CS. Then we used CIBERSORT algorithm to observe the differences in the distribution of 28 types of immune cells. Thus, we assessed the relationship between CS expression and tumor infiltrating immune cells (TIICs).

\subsection{An analysis of PPI network and its related module}

To construct the correlation network diagram, we identified top 50 positive and negative co-expressed genes from STRING database. Then these genes were visualized with Cytoscape software(version 3.8.2.(17) Subsequently, molecular complex detection algorithm was used to find the two protein modules that interacted with each other.

\subsection{Statistical analysis}

We obtained CS related data from TCGA database, GEO database and used R software (version4.0.3) for further processing. UALCAN database, LinkedOmics database, TIMER database, GEPIA Database and Cibersort website were used to analyze the relationship between CS and clinical traits of LUAD patients as well as the relationship between CS and TIICs. Immunohistochemical samples were collected from The Affiliated Hospital of Nantong University. All the above data, $\mathrm{P}<0.05$ was considered statistically significant.

\section{Declarations}

\section{Disclosure Statement}

The authors declared that there is no conflict of interest

\section{The Authors' Contributions}

RF conceived the study designed, conducted the statistical analyses, and provided significant input in the writing of the manuscript. TTB and SSH participated in the study design and statistical analyses. All authors provided assistance in the statistical analyses. JL and YFL revised the manuscript and approved the final manuscript.

\section{Acknowledgement}

The author would like to thank TCGA and GEO, TIMER, Kaplan-Meier Plotter, Ualcan, LinkedOmics databases for providing the data in this study. 


\section{Founding}

This study was funded by grants from Nantong Municipal Science and technology project (No. MSZ19164, JCZ0001), Jiangsu Post-doctoral Foundation Research Project, China (No. 2019Z142), Key Talents of Medical Science in Jiangsu Province, China (No. QNRC2016682), Scientific Research Project of Nantong Municipal Health Commission (Grant. No. QA2019060) and Postgraduate Research and Practice Innovation Program of Jiangsu province (No. SJCX20-1165, SJCX21-1473).

\section{Availability of data and materials}

The data results supporting this study come from TCGA database (https://genome-cancer.ucsc.edu/) $\triangle T$ IMER

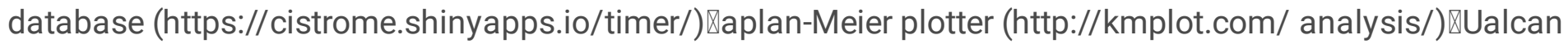
(http://ualcan.path.uab.edu/index.html)囚LinkedOmics (http://www.linkedomics.org/login.php).

\section{Ethics approval and consent to participate}

The authors state that the human sample was reviewed and approved by the Institutional Review Committee of Affiliated Hospital of Nantong University (review number: 2021-L021)

\section{Consent for publication}

All presentations of case reports have consent for publication.

\section{References}

1. Bade BC, Dela Cruz CS. Lung Cancer 2020: Epidemiology, Etiology, and Prevention. Clin Chest Med. 2020;41(1):1-24.

2. Goldstraw P, Ball D, Jett JR, Le Chevalier T, Lim E, Nicholson AG, et al. Non-small-cell lung cancer. Lancet. 2011;378(9804):1727-40.

3. Cancer Genome Atlas Research N. Comprehensive molecular profiling of lung adenocarcinoma. Nature. 2014;511(7511):543-50.

4. Sepesi B, Cascone T, Chun SG, Altan M, Le X. Emerging Therapies in Thoracic MalignanciesImmunotherapy, Targeted Therapy, and T-Cell Therapy in Non-Small Cell Lung Cancer. Surg Oncol Clin N Am. 2020;29(4):555-69.

5. Arneth B. Tumor Microenvironment. Medicina (Kaunas). 2019;56(1).

6. Suissa M, Suda K, Schatz G. Isolation of the nuclear yeast genes for citrate synthase and fifteen other mitochondrial proteins by a new screening method. EMBO J. 1984;3(8):1773-81.

7. Schlichtholz B, Turyn J, Goyke E, Biernacki M, Jaskiewicz K, Sledzinski Z, et al. Enhanced citrate synthase activity in human pancreatic cancer. Pancreas. 2005;30(2):99-104.

8. Anderson AS, Roberts PC, Frisard MI, McMillan RP, Brown TJ, Lawless MH, et al. Metabolic changes during ovarian cancer progression as targets for sphingosine treatment. Exp Cell Res. 2013;319(10):1431-42. 
9. Lin CC, Cheng TL, Tsai WH, Tsai HJ, Hu KH, Chang HC, et al. Loss of the respiratory enzyme citrate synthase directly links the Warburg effect to tumor malignancy. Sci Rep. 2012;2:785.

10. Cai Z, Deng Y, Ye J, Zhuo Y, Liu Z, Liang Y, et al. Aberrant Expression of Citrate Synthase is Linked to Disease Progression and Clinical Outcome in Prostate Cancer. Cancer Manag Res. 2020;12:6149-63.

11. Li YW, Qiu SJ, Fan J, Zhou J, Gao Q, Xiao YS, et al. Intratumoral neutrophils: a poor prognostic factor for hepatocellular carcinoma following resection. J Hepatol. 2011;54(3):497-505.

12. Shitara K, Nishikawa H. Regulatory T cells: a potential target in cancer immunotherapy. Ann N Y Acad Sci. 2018;1417(1):104-15.

13. Steidl C, Lee T, Shah SP, Farinha P, Han G, Nayar T, et al. Tumor-associated macrophages and survival in classic Hodgkin's lymphoma. N Engl J Med. 2010;362(10):875-85.

14. Li T, Fan J, Wang B, Traugh N, Chen Q, Liu JS, et al. TIMER: A Web Server for Comprehensive Analysis of Tumor-Infiltrating Immune Cells. Cancer Res. 2017;77(21):e108-e10.

15. Lanczky A, Nagy A, Bottai G, Munkacsy G, Szabo A, Santarpia L, et al. miRpower: a web-tool to validate survival-associated miRNAs utilizing expression data from 2178 breast cancer patients. Breast Cancer Res Treat. 2016;160(3):439-46.

16. Gentles AJ, Newman AM, Liu CL, Bratman SV, Feng W, Kim D, et al. The prognostic landscape of genes and infiltrating immune cells across human cancers. Nat Med. 2015;21(8):938-45.

17. Shannon P, Markiel A, Ozier O, Baliga NS, Wang JT, Ramage D, et al. Cytoscape: a software environment for integrated models of biomolecular interaction networks. Genome Res. 2003;13(11):2498-504.

18. Martin-Sanchez JC, Lunet N, Gonzalez-Marron A, Lidon-Moyano C, Matilla-Santander N, Cleries R, et al. Projections in Breast and Lung Cancer Mortality among Women: A Bayesian Analysis of 52 Countries Worldwide. Cancer Res. 2018;78(15):4436-42.

19. Cardaci S, Ciriolo MR. TCA Cycle Defects and Cancer: When Metabolism Tunes Redox State. Int J Cell Biol. 2012;2012:161837.

20. Gaude E, Frezza C. Defects in mitochondrial metabolism and cancer. Cancer Metab. 2014;2:10.

21. Chen L, Liu T, Zhou J, Wang Y, Wang X, Di W, et al. Citrate synthase expression affects tumor phenotype and drug resistance in human ovarian carcinoma. PLoS One. 2014;9(12):e115708.

22. Simonnet H, Alazard N, Pfeiffer K, Gallou C, Beroud C, Demont J, et al. Low mitochondrial respiratory chain content correlates with tumor aggressiveness in renal cell carcinoma. Carcinogenesis. 2002;23(5):759-68.

23. Remark R, Becker C, Gomez JE, Damotte D, Dieu-Nosjean MC, Sautes-Fridman C, et al. The non-small cell lung cancer immune contexture. A major determinant of tumor characteristics and patient outcome. Am J Respir Crit Care Med. 2015;191(4):377-90.

24. Harjunpaa H, Llort Asens M, Guenther C, Fagerholm SC. Cell Adhesion Molecules and Their Roles and Regulation in the Immune and Tumor Microenvironment. Front Immunol. 2019;10:1078.

25. Takeuchi Y, Nishikawa H. Roles of regulatory T cells in cancer immunity. Int Immunol. 2016;28(8):401-9.

26. Yuen GJ, Demissie E, Pillai S. B lymphocytes and cancer: a love-hate relationship. Trends Cancer. 2016;2(12):747-57. 
27. Banat GA, Tretyn A, Pullamsetti SS, Wilhelm J, Weigert A, Olesch C, et al. Immune and Inflammatory Cell Composition of Human Lung Cancer Stroma. PLoS One. 2015;10(9):e0139073.

28. Kodama M, Kodama T, Newberg JY, Katayama H, Kobayashi M, Hanash SM, et al. In vivo loss-of-function screens identify KPNB1 as a new druggable oncogene in epithelial ovarian cancer. Proc Natl Acad Sci U S A. 2017;114(35):E7301-E10.

29. Wang X, Chang X, He C, Fan Z, Yu Z, Yu B, et al. ATP5B promotes the metastasis and growth of gastric cancer by activating the FAK/AKT/MMP2 pathway. FASEB J. 2021;35(4):e20649.

30. Xu A, Shang W, Wang Y, Sun X, Zhou B, Xie Y, et al. ALA protects against ERS-mediated apoptosis in a cochlear cell model with low citrate synthase expression. Arch Biochem Biophys. 2020;688:108402.

\section{Figures}

\section{Figure 1}

Caption not included with this version. 
A

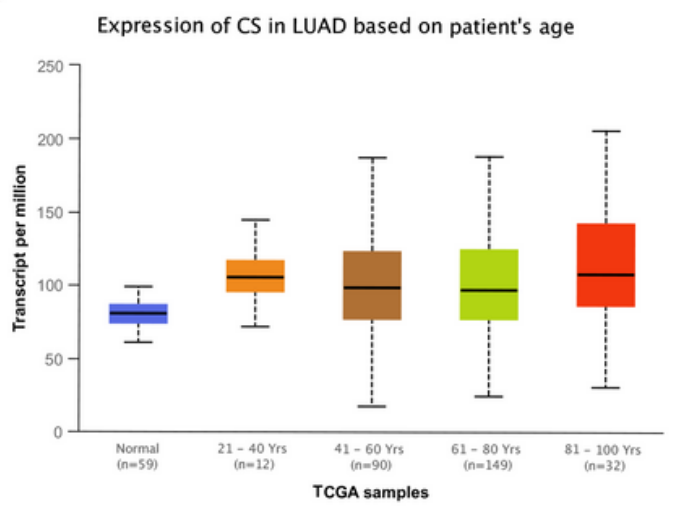

C

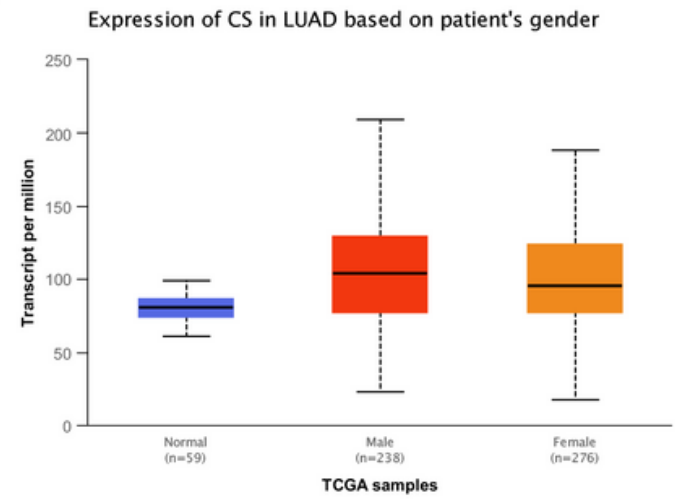

E

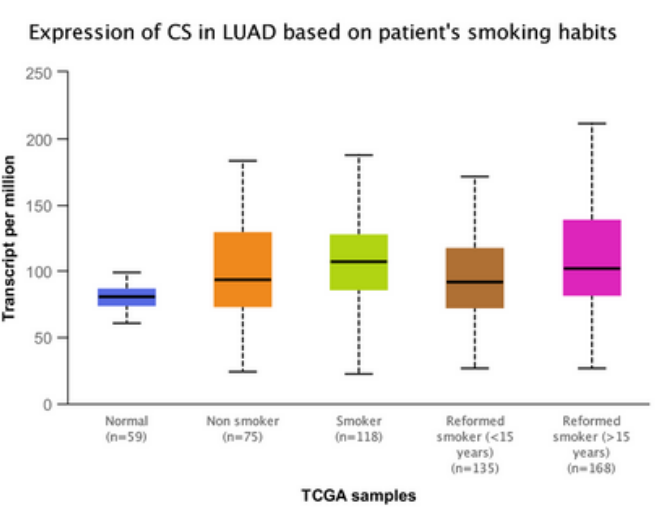

B

Expression of CS in LUAD based on nodal metastasis status

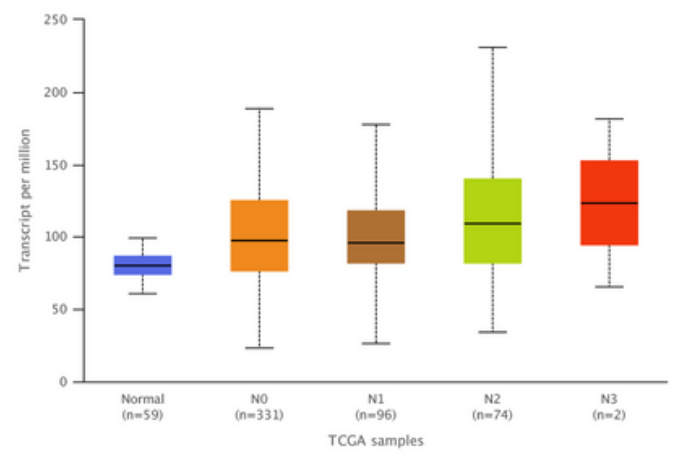

D

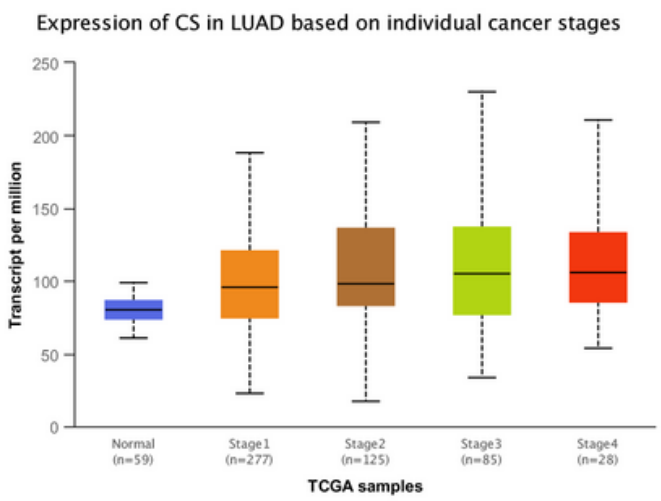

$\mathrm{F}$

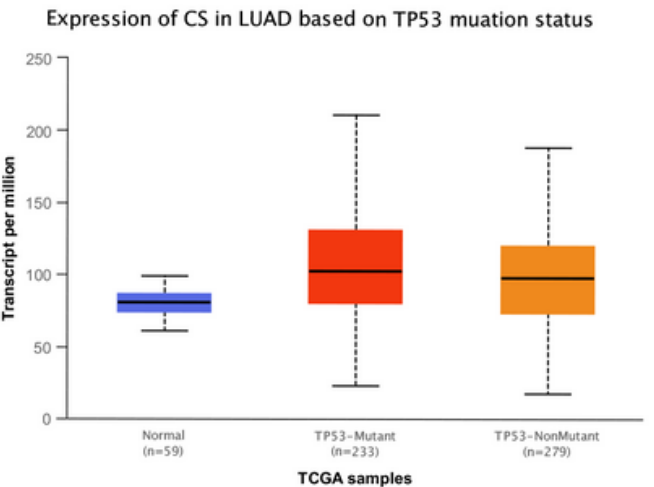

Figure 2

Caption not included with this version.

Figure 3

Caption not included with this version. 


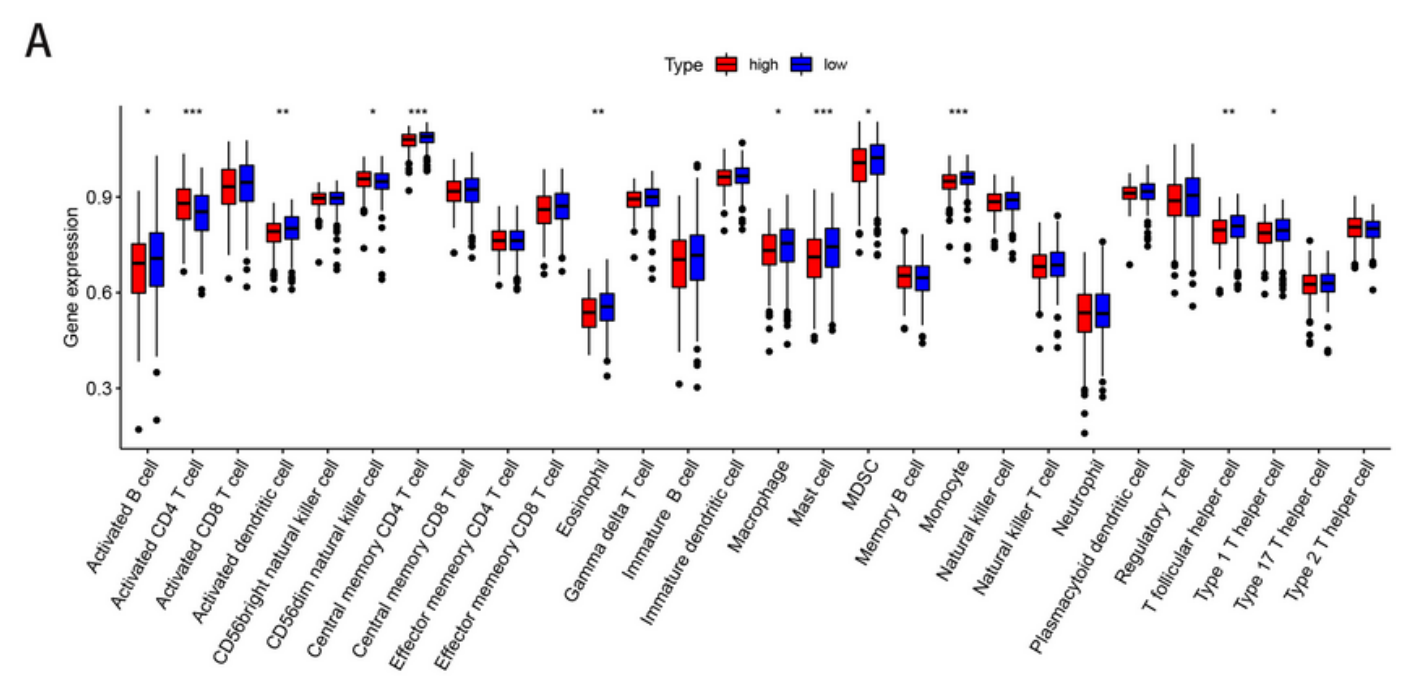

B

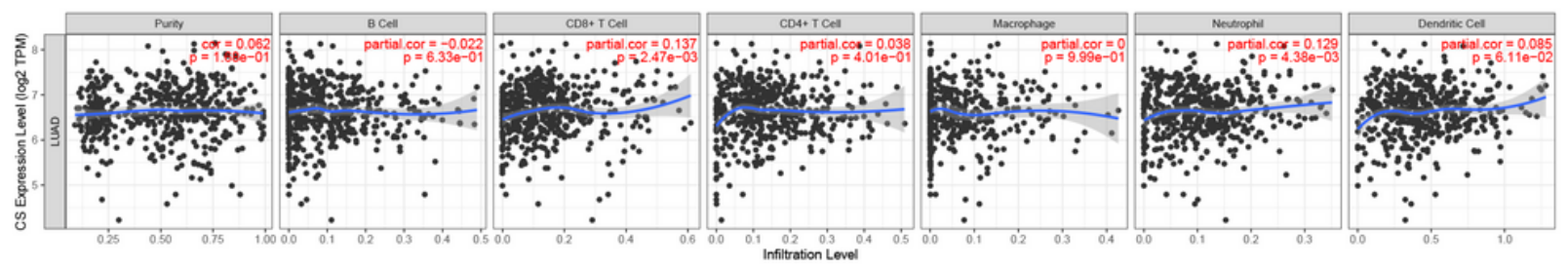

C

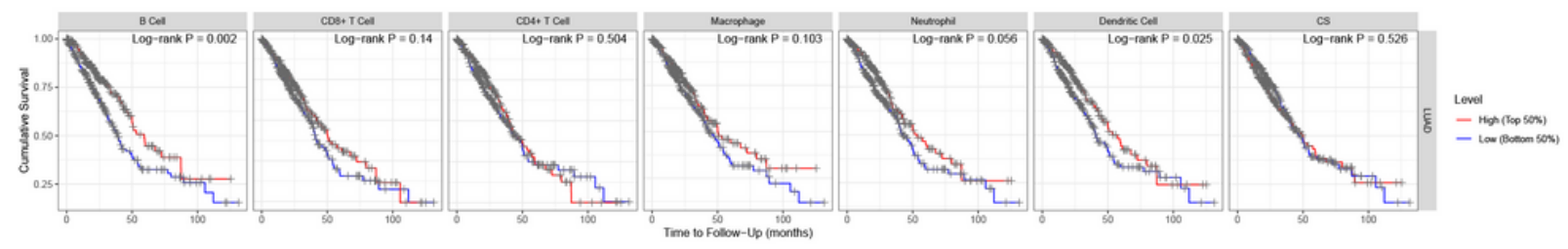

Figure 4

Caption not included with this version. 
A

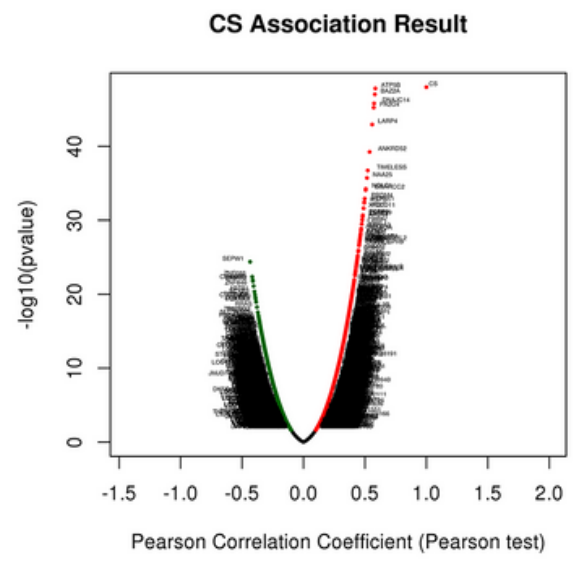

B

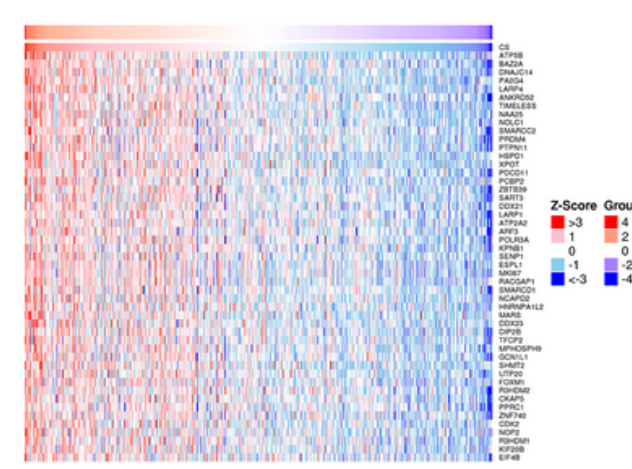

C

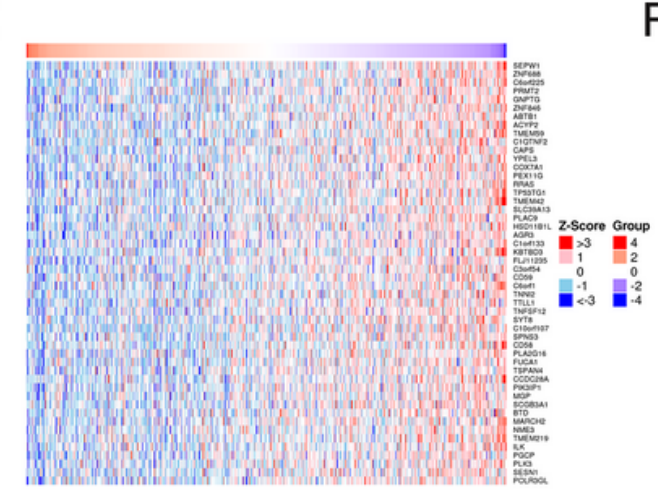

E
D
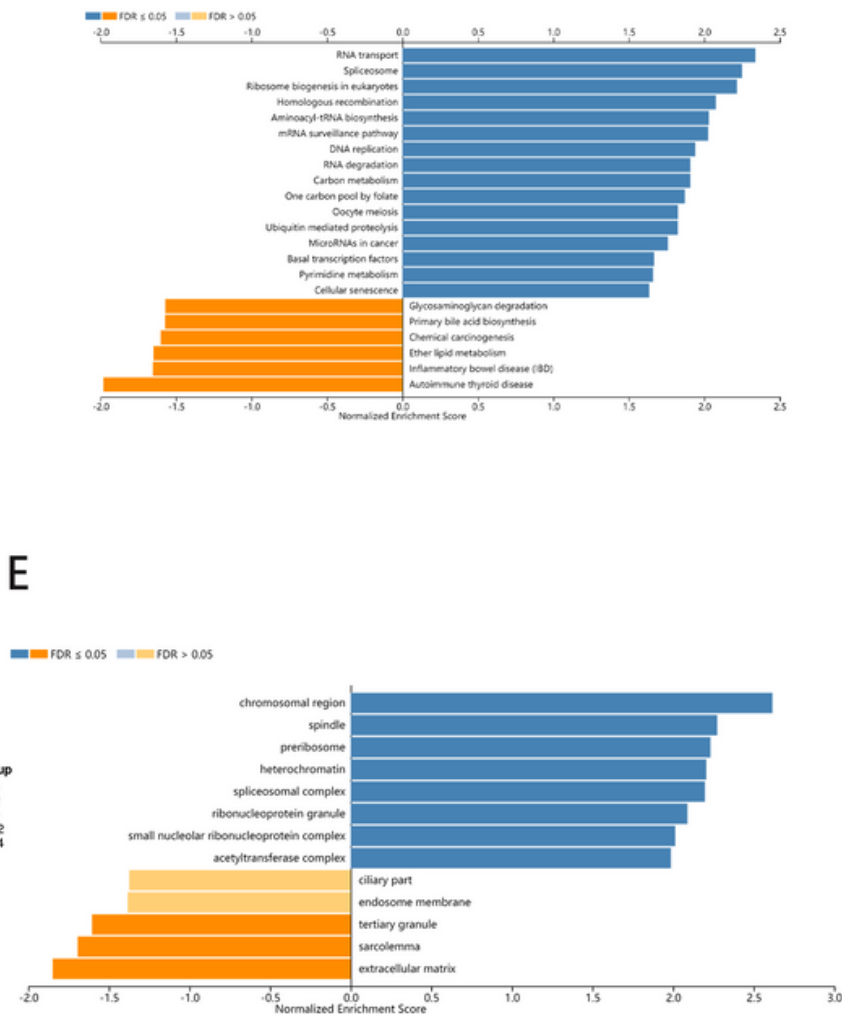

F

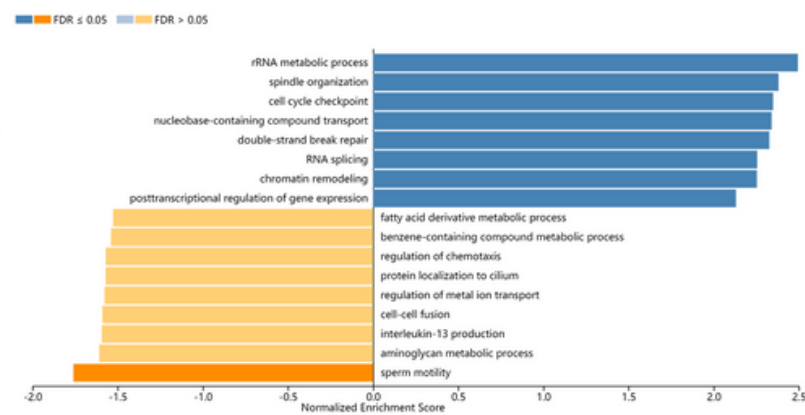

Figure 5

Caption not included with this version.

\section{Figure 6}

Caption not included with this version. 
Figure 7

Caption not included with this version.

Page 21/21 